\title{
Office and laboratory blood pressures as predictors of daily blood pressure level in normotensive subjects and borderline and mild hypertensive subjects
}

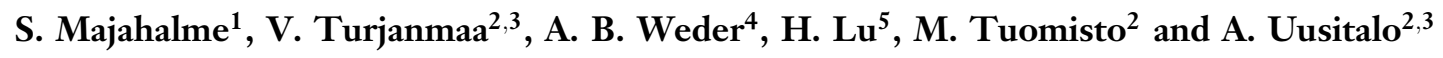 \\ Departments of ${ }^{1}$ Medicine and ${ }^{2}$ Clinical Physiology, Medical School, University of Tampere, Tampere, Finland \\ ${ }^{3}$ Department Clinical Physiology, Tampere University Hospital, Tampere, Finland \\ ${ }^{4}$ Department of Internal Medicine, Division of Hypertension, University of Michigan, Ann Arbor, USA ${ }^{5}$ Center of Drug Evaluation and Research, Office of \\ Biometrics and Epidemiology, Food and Drug Administration, Rockville, MD, USA \\ Received 28 June 1997; accepted 12 December 1997 \\ Correspondence: S. Majahalme, Tampere University Hospital, Department of Internal Medicine/Cardiology, PO Box 2000, 33521 Tampere, Finland
}

\section{Summary}

A series of standardized laboratory tests [10 min sitting and supine, 9 min standing, dynamic; cycle ergometer (ERG) and isometric exercise; handgrip (HG)] were performed during intra-arterial blood pressure (BP) recording in 97 healthy unmedicated men, initially classified as normotensive (NT, $n=34)$, borderline hypertensive (BHT, $n=29$ ) or mildly hypertensive (HT, $n=34$ ) by repeated office blood pressure (OBP) measurements. After testing, a 24-h intra-arterial ambulatory BP (IABP) recording was obtained while subjects performed their normal activities. Day and night periods were analysed as well as 24-h averages for systolic BP (SBP) and diastolic BP (DBP) using Pearson correlations and multiple linear regressions. In normotensive subjects, the supine SBP predicted IABP measurements best $(r$ range 0.39-0.69, $P<0 \cdot 05-$ 0.001). In multiple regression, supine SBP explained $49 \%$ of $24-\mathrm{h} \mathrm{SBP}$ variance $(F=12 \cdot 4, P=0 \cdot 001)$. For BHT, supine SBP was also the best predictor $(r$ range $0.09-0.64, P$ NS to $P<0 \cdot 001$ ), and it explained $37 \%$ of 24-h SBP variance $(F=15 \cdot 6, P=0 \cdot 0005)$. In HT, ERG DBP correlated best with IABP ( $r$ range $0.52-$ $0 \cdot 75, P<0 \cdot 01-0 \cdot 001)$. ERG SBP explained $49 \%$ of $24-\mathrm{h}$ SBP $(F=31 \cdot 0, P=0 \cdot 0000)$ and ERG DBP explained $56 \%$ of $24-\mathrm{h}$ DBP $(F=35 \cdot 4, P=0 \cdot 0000)$ variance. Laboratory BP correlations were generally better with day than with night measurements. OSBP correlated moderately well with IABP in NT, and weakly in BHT and HT; ODBP instead correlated with IABP in NT and HT but not significantly in BHT. In conclusion, OBP is less closely related to IABP than laboratory BP, but even laboratory BP generally explains less than $50 \%$ of IABP variance. Stressors such as exercise are useful only in HT. For BHT, the prediction of IABP with laboratory measures was even weaker than in other groups, and thus ambulatory measurements cannot be replaced by short-duration laboratory measurements and stress tests.

Keywords: ambulatory BP, exercise BP, intra-arterial $\mathrm{BP}$, postural BP.

\section{Introduction}

Many studies have shown that office blood pressure (OBP) predicts cardiovascular events and targets organ damage in hypertension (Bulpitt et al., 1992; McCloskey et al., 1992; Kannel, 1993; Lauer et al., 1993), and evidence is accumulating that ambulatory blood pressure (ABP) may improve the prediction of target organ damage (Pickering et al., 1983; Devereux \& Pickering, 1988; Prisant \& Carr, 1990; Verdecchia et al., 1990a,b; Rizzoni et al., 1992) and cardiovascular events related to hypertension. (Perloff et al., 1983, 1989, 1991; 
Pickering \& Devereux, 1987; Frattola et al., 1993; Verdecchia et al., 1994). However, ambulatory recording is time-consuming, requires greater patient compliance and is more expensive than an average clinical visit for blood pressure (BP) measurement.

With repeated office BP measurements Pearce et al. (1992) have shown very high correlations between office and ambulatory measurements in white, elderly men, and also reported a low incidence of white-coat hypertension, which is one of the recommended indications for ambulatory recording. Pickering et al. (1982) have shown in a population of normotensive subjects, borderline hypertensive subjects and hypertensive subjects that office readings gave good predictions of the average 24-h pressure in normotensive subjects and established hypertensive subjects, but not in borderline hypertensive subjects.

Another strategy for estimating average ambulatory $\mathrm{BP}$ and thus daily BP level is to use dynamic measures. Combined postural measurements have been shown to predict 24-h ABP in hypertensive subjects (Zachariah et al., 1990), as have BP responses to psychological laboratory tasks (Morales-Ballejo et al., 1988). Harshfield et al. (1988) studied untreated mild hypertensive subjects using both active coping tasks and dynamic exercise to predict daily BP, and they demonstrated that achieved BP during active coping tasks correlated better than achieved BP during the exercise test with ambulatory pressure, but $\mathrm{BP}$ change during tasks correlated only weakly, if at all.

Our aim was to study the usefulness of BP measurements during standardized postural and exercise tests in the prediction of daily $\mathrm{BP}$ level compared with the mean of repeated office measurements. Our study examined normotensive subjects and both borderline hypertensive subjects and mild hypertensive subjects, and to get the best possible accuracy for the BP measurements we used intraarterial recording for both laboratory and ambulatory measurements.

\section{Methods}

\section{Subjects}

Ninety-seven healthy male volunteers were recruited from a routine health check-up carried out on all 35-, 40- and 45-year-old people at the Communal
Health Centre in the city of Tampere. Detailed protocols for recruitment and for blood pressure measurements are described in an earlier publication (Majahalme et al., 1996). Subjects were classified according to WHO criteria (WHO Expert Committee, 1978) based on the mean of repeated office BP measurements obtained before the trial, and included 34 normotensive subjects [NT, systolic $\mathrm{BP}(\mathrm{SBP}) \leq 140$ and diastolic $\mathrm{BP}(\mathrm{DBP}) \leq 90$ ], 29 borderline hypertensive subjects (BHT, SBP $141-159 \mathrm{mmHg}$ or DBP $91-94 \mathrm{mmHg}$ ) and 34 mild hypertensive subjects (HT, SBP $\geq 160$ or DBP $\geq 95 \mathrm{mmHg}$ ). Classification blood pressures and demographic characteristics of the subjects are shown in Table 1. Clinical examination, chest radiography, electrocardiography and screening haematology and serum biochemistry tests were normal in each subject. No subjects had ever taken antihypertensive medication or other chronic medications. Alcoholic beverages were prohibited 2 days before and during the study, and consumption of food, coffee or tobacco was not allowed during the laboratory test period.

The study was approved by the ethics committee of Tampere University Hospital. Written informed consent was obtained before the study.

Table 1 Characteristics of study population.

\begin{tabular}{|c|c|c|c|}
\hline & NT $(n=34)$ & BHT $(n=29)$ & HT $(n=34)$ \\
\hline Age (years) & $39 \cdot 6(4 \cdot 1)$ & $40.9(4 \cdot 0)$ & $39 \cdot 8(3 \cdot 8)$ \\
\hline Weight (kg) & $81.0(8.5)$ & $84 \cdot 1(9 \cdot 5)$ & $83 \cdot 8(9 \cdot 6)$ \\
\hline Height $(\mathrm{cm})$ & $179 \cdot 5(6 \cdot 4)$ & $181 \cdot 0(7 \cdot 3)$ & $177 \cdot 0(4 \cdot 9)$ \\
\hline $\mathrm{BSA}\left(\mathrm{m}^{2}\right)$ & $2 \cdot 0(0.1)$ & $2 \cdot 1(0 \cdot 1)$ & $2 \cdot 1(0 \cdot 1)$ \\
\hline BMI $\left(\mathrm{kg} \mathrm{m}^{-2}\right)$ & $25(2 \cdot 8)$ & $26(3 \cdot 1)$ & $27(2 \cdot 6)$ \\
\hline Skinfold index (\%) & $25(5 \cdot 6)$ & $25(5 \cdot 0)$ & $25(4 \cdot 4)$ \\
\hline \multicolumn{4}{|l|}{ Classification (cuff) } \\
\hline $\mathrm{SBP}(\mathrm{mmHg})$ & $131(6 \cdot 7)$ & $143(4 \cdot 9) \ddagger$ & $153(9 \cdot 8) \dagger$ \\
\hline $\mathrm{DBP}(\mathrm{mmHg})$ & $82(5 \cdot 3)$ & $90(4 \cdot 0) \dagger$ & $99(5 \cdot 5) \neq$ \\
\hline ERG max load (W) & $195(33.9)$ & $193(35 \cdot 3)$ & $178(28.0)$ \\
\hline Hand grip max load & $120(17.9)$ & $118(16 \cdot 6)$ & $119(17 \cdot 5)$ \\
\hline
\end{tabular}

NT, normotensive group; BHT, borderline hypertensive group; $\mathrm{HT}$, mildly hypertensive group; BSA, body surface area; BMI, body mass index; ERG, cycle dynamic exercise test. ANOVA, $₫ P<0.001$. 


\section{Blood pressure measurement}

\section{Office measurements}

OBP used in classifying and in comparisons was measured during a 2-month period before the intra-arterial study using a standard cuff and sphygmomanometer. Measurements took place in the doctor's office in most cases between $10.00 \mathrm{~h}$ and $15.00 \mathrm{~h}$. No recommendations of diet or smoking were given before these measurements. All measurements were performed in the sitting position after at least $5 \mathrm{~min}$ of resting. Phase I of Korotkoff's sounds was used to determine the systolic pressure and phase $\mathrm{V}$ to determine the diastolic pressure. In each session three different readings, each at least $1 \mathrm{~min}$ apart, were taken, and the mean (total of six to nine readings) was used to calculate the classification blood pressure. In addition, we also implied that the mean BP of two consecutive reading sessions should belong to the same WHO category to allow the subject to be classified.

The mean of repeated OBP was also used in all comparisons with intra-arterial BP in different situations.

To record intra-arterial $\mathrm{BP}$, we used the Oxford method (as described in detail elsewhere; Kalli, 1984; Takalo et al., 1994) during a test series of postural changes and exercise as well as throughout a 24-h ambulatory period (IABP). The brachial artery of the non-dominant arm was cannulated using the Seldinger technique under local anaesthesia, and signals were collected on Medilog 20 FM recorders (Oxford Medical Systems, Abingdon, Oxford, UK). The system for signal analysis has been described and validated in previous studies (Kalli et al., 1985; Turjanmaa, 1989). After processing, 30-s means of BP were used for calculations.

\section{Diurnal BP level}

The mean intra-arterial $24-\mathrm{h}$ period as well as the means of two 5-h segments, day (Day, 08.30-13.30 h) representing the working period, and night (Night, $00.30-17.30 \mathrm{~h}$ ) representing the sleeping period, were used for comparisons.

\section{Laboratory testing}

Laboratory tests included postural and exercise tests.

\section{Postural tests}

Sitting (SIT) and supine (SUP) testing periods each lasted $10 \mathrm{~min}$, during which the subjects were awake but not allowed to speak. A third test period of standing (STA) lasted 9 min during which subjects were asked to stand comfortably without moving or speaking. Postural testing was performed in this order on every occasion. The time of the test was marked on the BP signal tape with a special event marker prepared for these purposes (Kalli, 1984; Turjanmaa et al., 1991) and was double checked with a clock. To evaluate the effect of each position on BP, we used the mean $\mathrm{BP}$ of the final min of each test.

\section{Dynamic exercise testing}

Dynamic exercise testing (ERG) was performed in an upright position using a cycle ergometer (Siemens Elema, Germany). The starting workload was $50 \mathrm{~W}$, and the workload was increased in a stepwise manner with increments of $50 \mathrm{~W}$ every 4 min until the subject achieved a heart rate of at least 160 beats $\min ^{-1}$. The mean value of $\mathrm{BP}$ during the final minute of each workload was used for comparisons.

\section{A submaximal handgrip test}

A submaximal handgrip test (HG) was used as an isometric exercise. It was performed using a Vigorimeter (Martin, Germany) at 30\% of the subject's maximal effort, determined as a mean of three maximal contractions performed a day before the test period. Submaximal gripping lasted as long as the subject could do it or for a maximum of $5 \mathrm{~min}$. Subjects were prevented from performing Valsalva's manoeuvre because the investigator was talking with the subject during the test. The mean value of $\mathrm{BP}$ during the final minute of the test was used for comparisons.

\section{Testing protocol}

The morning of the study the subjects ate a standardized light breakfast before $07.30 \mathrm{~h}$. The 
IABP system was attached and calibrated as described by Turjanmaa (1989) in the morning between $08.00 \mathrm{~h}$ and $09.00 \mathrm{~h}$. Subjects were then tested in a quiet laboratory. Postural and exercise tests started in every case after $11.00 \mathrm{~h}$ The first posture was SIT, which was followed by isometric exercise, and after an interlude SUP and STA positions were performed. Dynamic exercise was the last test in the protocol after which a 10 -min supine rest period followed. All tests were completed before $14.00 \mathrm{~h}$ in every case. The ambulatory period started after $14.00 \mathrm{~h}$, and for the next $24 \mathrm{~h}$ subjects lived in their normal environment and kept a detailed diary of their activities.

For statistical analysis, one-way analysis of variance (ANOVA) with multiple comparison tests of Tukey were used as regression analyses using SAS 6.09 Unix statistical software. Data are presented as means and SDs. Correlations were calculated as Pearson correlation coefficients. $P$-values less than 0.05 were considered significant.

\section{Results}

The clinical characteristics of the population are shown in Table 1. No significant differences were seen in demographic variables. By design, there were clear differences in classification BP between groups (Table 1), but for postural and ambulatory BP, borderline hypertensive subjects were closer to hypertensive subjects than to normotensive subjects (Figs 1 and 2). No statistically significant differences were observed in achieved exercise workloads during dynamic and isometric exercise.

\section{Office BP, laboratory BP and ambulatory BP}

Correlations between 24-h IABP levels and office, laboratory, and Day and Night BP levels are shown in Tables 2 and 3. Normotensive subjects and hypertensive subjects had somewhat better correlations between OBP and 24-h IABP than borderline hypertensive subjects.

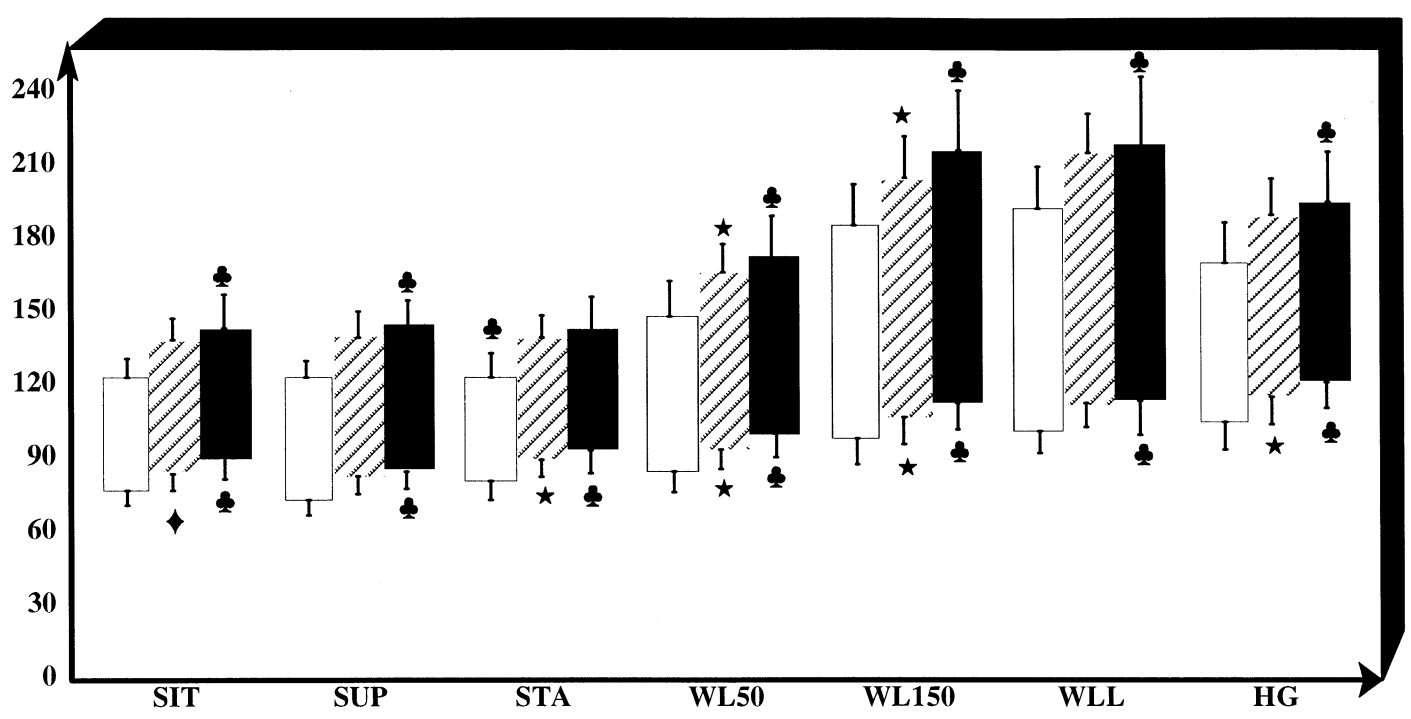

Classification

\begin{tabular}{|c|c|c|}
\hline NT $\square$ & ANOVA: & $\star=P<0.05$ \\
\hline BHT & & $\downarrow=P<0.01$ \\
\hline & & $=P<0.001$ \\
\hline
\end{tabular}

Figure $1 \mathrm{BP}$ and HR levels in different postures and during exercise. SIT and SUP, mean of final minute of 10 min sitting and supine; STA, mean of final minute of 9-min standing; WL50, $50 \mathrm{~W}$ workload; WL150, $150 \mathrm{~W}$ workload; WLL, last workload; HG, handgrip $30 \%$ of maximal effort; SBP, systolic blood pressure; DBP, diastolic blood pressure; HR, heart rate. 


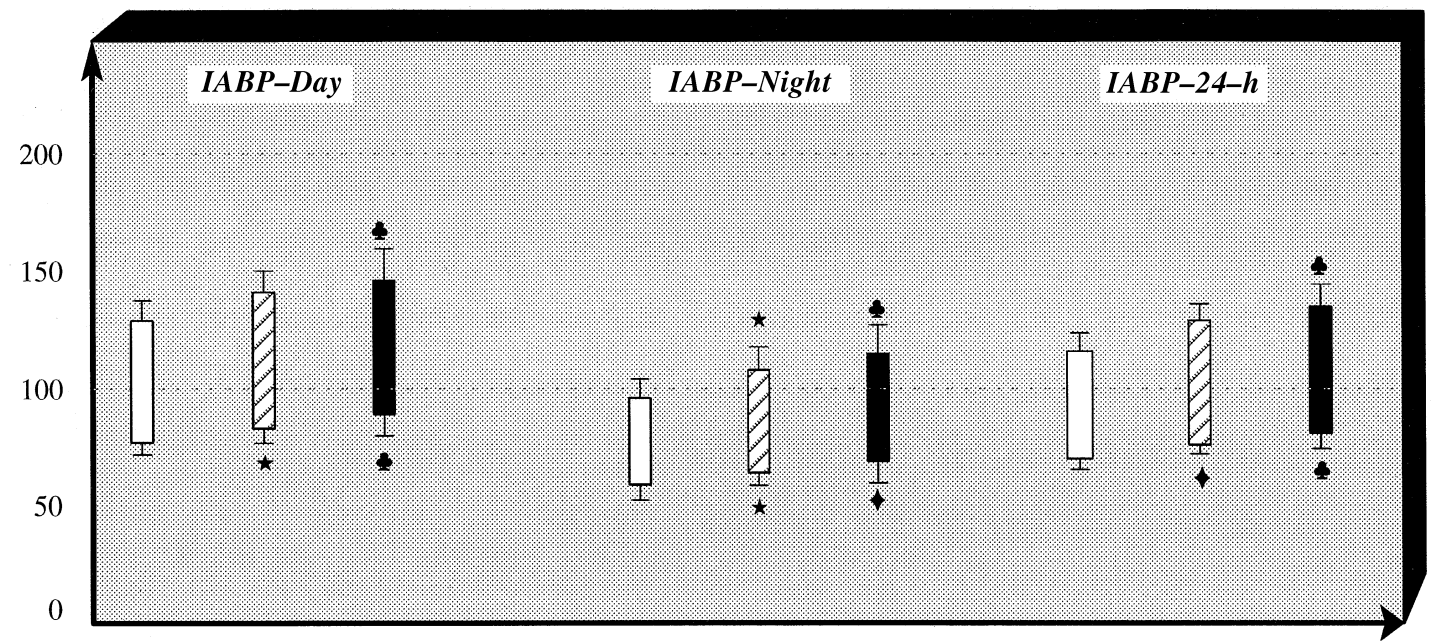

\section{Classification}

\begin{tabular}{|c|c|c|}
\hline NT $\square$ & ANOVA: & $\star=P<0.05$ \\
\hline BHT 2 & & $\downarrow=P<0.01$ \\
\hline & & $=P<0.001$ \\
\hline
\end{tabular}

Figure 2 Intra-arterial blood pressure levels in study population. NT, normotensive subjects; BHT, borderline hypertensive subjects; HT, mildly hypertensive subjects; SBP, systolic blood pressure, 5-h mean and 24-h mean; DBP, diastolic blood pressure, 5-hour mean and 24-h mean; HR, heart rate, 5-h mean and 24-h mean; $24 \mathrm{~h}$, mean of 24-h intra-arterial BP; DAY, mean of 5-h period during day; NIGHT, mean of 5 -h period during night.

Intra-arterial postural BP had generally higher correlations with ambulatory readings in NT and BHT compared with achieved BP during exercise, whereas in HT BP readings during dynamic exercise tended to have the best correlations. It was also apparent that during increasing workload correlations between ambulatory and exercise BP decreased in NT and BHT, whereas in HT the trend was the opposite.
Isometric exercise did not prove to be a very useful predictor of IABP level.

Tables 4-6 show the results of multiple linear regression analyses in NT, BHT and HT separately. The main finding is that for NT and HT laboratory $\mathrm{BP}$ readings were much better at predicting ambulatory BP than BHT. But even for NT and HT the value of different laboratory tests varied: for NT,

Table 2 Correlations between office, intra-arterial postural, exercise and day and night 5-h level of SBP with 24-h SBP level.

\begin{tabular}{|c|c|c|c|c|c|c|c|c|c|c|}
\hline \multirow[b]{2}{*}{ group } & \multirow[b]{2}{*}{ OBP } & \multicolumn{3}{|c|}{ Intra-arterial postures } & \multicolumn{4}{|c|}{ Intra-arterial exercise } & \multicolumn{2}{|c|}{ Intra-arterial 5-h leve } \\
\hline & & SIT & SUP & STA & WL50 & WL150 & WLL & HG & Day & Night \\
\hline NT & 0.58 & 0.63 & 0.69 & $0.60 \ddagger$ & 0.67 & $0.55 t$ & $0.52 \dagger$ & $0.50 \dagger$ & $0.71 \ddagger$ & $0 \cdot 70 \%$ \\
\hline BHT & $0.36^{*}$ & $0.53 \dagger$ & $0.64 \dagger$ & $0.41^{*}$ & 0.25 & 0.20 & 0.08 & 0.33 & 0.78 & $0.76+$ \\
\hline HT & $0.44^{*}$ & $0.48 \dagger$ & $0.57 \ddagger$ & $0.61 \ddagger$ & $0.59 \ddagger$ & $0 \cdot 70 \$$ & $0.67+$ & $0.52 \dagger$ & 0.84 & $0.80 \%$ \\
\hline ALL & $0.74 \ddagger$ & $0.71 末$ & $0.79 \$$ & $0.73 \%$ & $0.72 \ddagger$ & $0.70 \ddagger$ & $0.66 \ddagger$ & $0.64 末$ & $0.86 \ddagger$ & $0.86 \$$ \\
\hline
\end{tabular}

Simple Pearson correlation coefficients: ${ }^{\star} P<0.05 ; \uparrow P<0.01 ; \$ P<0.001$. NT, normotensive subjects; BHT, borderline hypertensive subjects; $\mathrm{HT}$, hypertensive subjects; ALL, all subjects analysed together $(n=97)$; SBP, systolic blood pressure; OBP, mean of six to nine office readings obtained during 2 months before the intra-arterial recording; SIT and SUP, mean of final minute of 10-min sitting and supine postures; STA, mean of final minute of 9-min standing; WL50, 50 W workload; WL150, $150 \mathrm{~W}$ workload; WLL, last workload; dynamic exercise; HG, isometric exercise (hand grip). 
Laboratory BP and daily BP level • S. Majahalme et al.

Table 3 Correlations between office, intra-arterial postural, exercise and day and night 5-h level of DBP with 24-h DBP level.

\begin{tabular}{|c|c|c|c|c|c|c|c|c|c|c|}
\hline \multirow[b]{2}{*}{ group } & \multirow[b]{2}{*}{ OBP } & \multicolumn{3}{|c|}{ Intra-arterial postures } & \multicolumn{4}{|c|}{ Intra-arterial exercise } & \multicolumn{2}{|c|}{ Intra-arterial 5-h level } \\
\hline & & SIT & SUP & STA & WL50 & WL150 & WLL & HG & Day & Night \\
\hline NT & $0.58^{y}$ & $0.77^{y}$ & $0.73 \%$ & $0.75 \%$ & $0.54 \%$ & $0.47 \dagger$ & $0.50 \dagger$ & $0 \cdot 44^{\star}$ & $0.67 \neq$ & $0.74 \hbar$ \\
\hline BHT & 0.29 & $0 \cdot 48^{y}$ & $0.56 \dagger$ & $0.50 \dagger$ & $0.38^{*}$ & $0.47^{*}$ & 0.36 & 0.35 & 0.79 & $0.46^{\star}$ \\
\hline HT & $0.44^{*}$ & $0 \cdot 49^{y}$ & $0.56 \dagger$ & $0.52 \dagger$ & $0.63 \%$ & $0.73 \ddagger$ & $0.75 \ddagger$ & $0.42^{*}$ & $0.81+$ & $0.89 \ddagger$ \\
\hline ALL & $0.75 \ddagger$ & $0.74^{y}$ & 0.76 & $0.74 \uparrow$ & 0.76 & $0.75 \ddagger$ & $0.71 \%$ & $0.65 \%$ & 0.68 & $0.82 \ddagger$ \\
\hline
\end{tabular}

Simple Pearson correlation coefficients: ${ }^{*} P<0 \cdot 05 ; \uparrow P<0.01 ; \ddagger P<0 \cdot 001$. DBP, diastolic blood pressure. For other abbreviations, see Table 2 .

Table 4 Linear multiple regressions for follow-up ambulatory blood pressure; model includes all intra-arterial and office variables.

\begin{tabular}{|c|c|c|c|c|c|c|c|c|}
\hline \multirow[b]{2}{*}{ Classification } & \multicolumn{4}{|c|}{ Dependent variable 24-h SBP } & \multicolumn{4}{|c|}{ Dependent variable 24-h DBP } \\
\hline & $\begin{array}{l}\text { Independent } \\
\text { variable }\end{array}$ & $R^{2}$ & $\boldsymbol{F}$ & $P$ & $\begin{array}{l}\text { Independent } \\
\text { variable }\end{array}$ & $R^{2}$ & $\boldsymbol{F}$ & $\boldsymbol{P}$ \\
\hline \multirow[t]{2}{*}{ NT } & (1) SUP SBP & $49 \%$ & $30 \cdot 4$ & $<0.001$ & (1) SIT DBP & $57 \%$ & $19 \cdot 1$ & $<0.001$ \\
\hline & (2) WL50 SBP adds & $11 \%$ & $9 \cdot 4$ & $<0.01$ & 2) WL150 DBP adds & $7 \%$ & $5 \cdot 7$ & $<0.05$ \\
\hline BHT & SUP SBP & $37 \%$ & $15 \cdot 6$ & $<0.001$ & SUP DBP & $26 \%$ & $9 \cdot 6$ & $<0.01$ \\
\hline \multirow[t]{2}{*}{$\mathrm{HT}$} & (1) WL150 SBP & $49 \%$ & $31 \cdot 0$ & $<0.001$ & (1) WLL DBP & $56 \%$ & $35 \cdot 4$ & $<0.001$ \\
\hline & & & & & (2) SIT DBP adds & $9 \%$ & $7 \cdot 7$ & $<0.01$ \\
\hline
\end{tabular}

NT, normotensive subjects; BHT, borderline hypertensive subjects; HT, hypertensive subjects; SBP, systolic blood pressure; DBP, diastolic blood pressure; $24 \mathrm{~h}$, mean of 24 -h intra-arterial recording. Other abbreviations see Table 2.

Table 5 Linear multiple regressions for intra-arterial ambulatory blood pressure, model includes all intra-arterial and office variables.

\begin{tabular}{|c|c|c|c|c|c|c|c|c|}
\hline \multirow[b]{2}{*}{ Classification } & \multicolumn{4}{|c|}{ Dependent variable 24-h SBP } & \multicolumn{4}{|c|}{ Dependent variable 24-h DBP } \\
\hline & $\begin{array}{l}\text { Independent } \\
\text { variable }\end{array}$ & $R^{2}$ & $\boldsymbol{F}$ & $P$ & $\begin{array}{l}\text { Independent } \\
\text { variable }\end{array}$ & $R^{2}$ & $\boldsymbol{F}$ & $P$ \\
\hline NT & SUP SBP & $21 \%$ & 8.7 & $<0.01$ & STA DBP & $33 \%$ & $16 \cdot 0$ & $<0.001$ \\
\hline \multirow[t]{2}{*}{$\mathrm{BHT}$} & (1) SUP SBP & $28 \%$ & $10 \cdot 7$ & $<0.01$ & STA DBP & $19 \%$ & $6 \cdot 5$ & $<0.05$ \\
\hline & (2) OBP SBP adds & $11 \%$ & $4 \cdot 8$ & $<0.05$ & & & & \\
\hline \multirow[t]{2}{*}{ HT } & (1) WL150 SBP & $45 \%$ & $25 \cdot 8$ & $<0.001$ & (1) WLL DBP & $45 \%$ & $21 \cdot 7$ & $<0.001$ \\
\hline & & & & & (2) SIT DBP adds & $13 \%$ & $9 \cdot 1$ & $<0.01$ \\
\hline
\end{tabular}

NT, normotensive subjects; BHT, borderline hypertensive subjects; HT, hypertensive subjects; SBP, systolic blood pressure; DBP, diastolic blood pressure; Day, mean of 5-hour period during day $(08.30 \mathrm{~h}$ to $13 \cdot 30 \mathrm{~h}$ ). For other abbreviations, see Table 2.

postural BP, generally supine, were superior to other laboratory or office BP values in explaining ambulatory $\mathrm{BP}$ values, whereas for $\mathrm{HT}$ exercise $\mathrm{BP}$ gave the best results.

\section{Discussion}

We studied a well-defined group of normotensive middle-aged men and newly diagnosed borderline and mildly hypertensive, non-medicated middle-aged men, who were recruited during a population-based health check-up. This is the first study of this kind of group comparing the usefulness of standardized laboratory postures and stressors vs. office measurements in the prediction of daily BP level. Our results showed that the prediction of daily BP did not exceed $50 \%$ with any single test, and particularly in borderline hypertensive subjects the prediction of daily $\mathrm{BP}$ 
S. Majahalme et al. • Laboratory BP and daily BP level

Table 6 Linear multiple regressions for follow-up ambulatory blood pressure, model includes all intra-arterial and office variables.

\begin{tabular}{|c|c|c|c|c|c|c|c|c|}
\hline \multirow[b]{2}{*}{ Classification } & \multicolumn{4}{|c|}{ Dependent variable 24-h SBP } & \multicolumn{4}{|c|}{ Dependent variable 24-h DBP } \\
\hline & $\begin{array}{l}\text { Independent } \\
\text { variable }\end{array}$ & $R^{2}$ & $\boldsymbol{F}$ & $P$ & $\begin{array}{c}\text { Independent } \\
\text { variable }\end{array}$ & $R^{2}$ & $\boldsymbol{F}$ & $\boldsymbol{P}$ \\
\hline \multirow[t]{2}{*}{ NT } & (1) SUP SBP & $34 \%$ & $16 \cdot 3$ & $<0.001$ & SUP DBP & $41 \%$ & $21 \cdot 9$ & $<0.001$ \\
\hline & (2) WLL SBP adds & $8 \%$ & $4 \cdot 4$ & $<0.05$ & & & & \\
\hline BHT & SUP SBP & $14 \%$ & $4 \cdot 4$ & $<0.05$ & \multicolumn{4}{|c|}{ No statistically significant findings } \\
\hline \multirow[t]{2}{*}{ HT } & (1) WL150 SBP & $15 \%$ & $5 \cdot 7$ & $<0.05$ & (1) WL150 DBP & $31 \%$ & $10 \cdot 3$ & $<0.01$ \\
\hline & & & & & (2) SIT DBP adds & $12 \%$ & $6 \cdot 3$ & $<0.01$ \\
\hline
\end{tabular}

NT, normotensive subjects; BHT, borderline hypertensive subjects; HT, hypertensive subjects; SBP, systolic blood pressure; DBP, diastolic blood pressure; Night, mean of 5-hour period during the night (00:30-5·30 h). For other abbreviations, see Table 2.

was poor. This finding parallels the results of Pickering et al. (1982), who studied 75 middle-aged people, divided into equal size groups of normotensive, borderline hypertensive and hypertensive subjects. Although only comparing office BP with ambulatory BP, they showed that normotensive subjects and established hypertensive subjects had good correlations between office BP and ambulatory $\mathrm{BP}$, whereas in borderline hypertensive subjects correlations were poor. The authors felt the result was explained by an exaggerated office BP response in many BHT subjects that would have caused many borderline subjects to be labelled as hypertensive subjects during normal screening. Because activity is the major component affecting daily $\mathrm{BP}$ variability (Clark et al., 1986; Turjanmaa et al., 1987; Baumgart et al., 1989; Chau et al., 1989; Pieper et al., 1993; Bursztyn et al., 1994; Veerman et al., 1995), office measurements generally cannot reflect daily BP closely. Borderline hypertensive subjects are also often considered to be hyper-reactive (Julius et al., 1986), which could further decrease the predictability value of office measurements.

In contrast, Pearce et al. (1992), who studied 50 elderly, white men from a population-based sample, found a very high correlation between repeated office BP and 24-h ambulatory recording. However, they used five separate office visits to get the mean office $\mathrm{BP}$ and compared that with one $24-\mathrm{h}$ recording. In this particular case, the cost-effectiveness and patient friendliness of office BP measurements over ambulatory BP may be questioned. They also had significantly higher ambulatory BP readings than office readings, which is not always the case. In our study, two to three office visits were used to obtain the mean office BP, which still correlated less than short-term laboratory measures with ambulatory pressures. One important difference compared with Pearce's study is that we used intra-arterial recording in our ambulatory studies, which may alter the correlations somewhat. The ambulatory intra-arterial method is accepted as a gold standard representing the closest possible true BP readings. By using intra-arterial recording we assumed that we could determine the potential benefit of laboratory and ambulatory measurements.

In our study, 5-h BP periods during the day and night were closely related to the 24-h BP level, paralleling the findings of Chanudet et al. (1992). However, the results were variable in systolic and diastolic pressures, and also between groups. Thus, although applicable, they are not truly representative, and one should carefully think about the indications and benefit of shorter ambulatory registrations.

We asked the question: could a simple standardized laboratory test, such as posture or exercise test improve the prediction of daily BP compared with office BP, and could these tests be used instead of ambulatory recording? For the first part of our question the result was variable: for normotensive subjects, correlations between office and ambulatory $\mathrm{BP}$ were close to laboratory test BP correlations, whereas for hypertensive subjects laboratory tests proved to correlate clearly better than office BP. In borderline hypertensive subjects, both repeated office and laboratory test BP were weak predictors of daily 
pressures. Harshfield et al. (1988) compared different laboratory tasks with ambulatory readings, but only in mild hypertensive subjects. In that population they found that BP during active coping tasks were better related to ambulatory BP ( $r$ range $0 \cdot 61-0 \cdot 76)$ than BP during treadmill exercise test ( $r$ range $0 \cdot 33-0 \cdot 45)$, but, in their study, office BP (mean of five measurements on the study morning) correlated as well as active coping tasks with ambulatory recording $(r$ range $0.71-$ $0 \cdot 74)$. Their findings of office $B P$ seem to be in disagreement with our findings in the hypertensive group, but at least some of the differences are because of the different methods used: in our study BP was measured intra-arterially during the whole study, whereas during tests Harsfield et al. (1988) used manual auscultation for BP measurement, which may be unreliable during exercise (Gould et al., 1985; Turjanmaa et al., 1988), and during 24-h recording they used an auscultatory (microphonic) ambulatory device. We know of no comparable studies of normotensive subjects and borderline hypertensive subjects addressing this topic.

In conclusion, standardized laboratory tests predict daily BP level better than repeated office BP readings, but, at best, predictability generally did not exceed $50 \%$. Predictive power varied from test to test between groups, although supine BP in normotensive subjects and borderline hypertensive subjects had closest correlations with the 24-h BP level. BP measurements during exercise test were of some use only in hypertensive subjects. Even with the most accurate BP measurement method, the daily BP prediction was only moderate, and thus short-duration laboratory tests or exercise cannot replace ambulatory recording in daily BP level estimation.

\section{Acknowledgements}

This work was financially supported by the Medical Research Fund of Tampere University Hospital and the Pirkanmaa fund of the Finnish Cultural Foundation. Mrs Pirjo Järventausta is highly appreciated for her technical assistance during the study.

\section{References}

Baumgart P., Walger P., Fuchs G., Dorst K. G., VetTER H. \& RAHN K. H. (1989) Twenty-four-hour blood pressure is not dependent on endogenous circadian rhythm. 7 Hypertens, 7, 331-334.

Bulpitt C. J., Palmer A. J., Fletcher A. E., et al. (1992) Relation between treated blood pressure and death from ischaemic heart disease at different ages: a report from the Department of Health Hypertension Care computing Project. 7 Hypertens, 10, 1273-1278.

Bursztyn M., Mekler J., Wachtel N. \& Ben-ishay D. (1994) Siesta and ambulatory blood pressure monitoring. Comparability of the afternoon nap and night sleep. Am 7 Hypertens, 7, 217-221.

Chanudet X., Chau N. P. \& Larroque P. (1992) Shortterm representatives of daytime and nigh-time ambulatory blood pressures. 7 Hypertens, 10, 595-600.

Chau P. N., Mallion J. M., de gaudemaris R., Ruche E., Siche J. P., Pelen O. \& Mathern G. (1989) Twentyfour-hour ambulatory blood pressure in shift workers. Circulation, 80, 341-347.

Clark L. A., Denby L., Harshfield G. A., Pickering T. G., Blank S. \& Laragh J. H. (1986) A quantitative analysis of the effects of activity and time of day on the diurnal variations of blood pressure. 7 Chron Dis, 40, 671681.

Devereux R. B. \& Pickering T. G. (1988) Relationship between ambulatory and exercise blood pressure and cardiac structure. Am Heart F, 116, 1124-1133.

Frattola A., Parati G., Cuspidi C., Albini F. \& Mancia G. (1993) Prognostic value of 24-hour blood pressure variability. 7 Hypertens, 11, 1133-1137.

Gould B. A., Hornung R. S., Altman D. G., Cashman P. M. M. \& RAFTERY E. B. (1985) Indirect measurement of blood pressure during exercise testing can be misleading. Br Heart 7, 53, 611-615.

Harshfield G. A., James G. D., Schlussel Y., Yee L. S., Blank S. G. \& Pickering T. G. (1988) Do laboratory tests of blood pressure reactivity predict blood pressure changes during everyday life? Am 7 Hypertens, 1, 168-174.

Julius S., Weder A. B. \& Hinderliter A. L. (1986) Does behaviorally induced blood pressure variability lead to hypertension? In: Handbook of Stress, Reactivity, and Cardiovascular Disease (eds Matthews, K., Weiss, S. M., Detre, T., Dembroski, T. M., Falkner, B., Manuck, S. B. \& Williams, R. B. Jr), Vol. 1, pp. 71-82. John Wiley \& Sons, New York.

Kalli S. (1984) Recording and Computerized Analysis of Ambulatory Long-Term Blood Pressure. Technical Research Centre of Finland, Tampere.

Kalli S., Järvilehto P., Näriäinen K., Saranummi N., Suoranta R., Turjanmaa V. \& Uusitalo A. (1985) Computerized analysis of long-term invasive blood pressure signals. In: Proceedings of the Fifth International Symposium on Ambulatory Monitoring (eds Dal Palu, C. \& Pessina, A. C.), pp. 93-98. Cleup Editore, Padua. 
KANNEL W. B. (1993) Hypertension as a risk factor for cardiac events - epidemiologic results of long-term studies. 7 Cardiovasc Pharmacol, 21, S27-S37.

Lauer R. M., Clarce W. R., Mahoney L. T. \& WitT J. (1993) Childhood predictors for high adult blood pressure. Ped Clin North Am, 40, 23-40.

Majahalme S., Turjanmaa V., Weder A. B., Lu H., Tuomisto M. T. \& Uusitalo A. (1996) Blood pressure level and variability in prediction of blood pressure after five-year follow-up. Hypertension, 28, 725-231.

McCloskey L. W., Psaty B. M., Koepsell T. D. \& AAGAARD G. N. (1992) Level of blood pressure and risk of myocardial infarction among treated hypertensive patients. Arch Intern Med, 152, 513-520.

Morales-ballejo H. M., Eliot R. S., Boone J. L. \& Hughes J. S. (1988) Psychophysiologic stress testing as a predictor of mean daily blood pressure. Am Heart 7, 116, 673-681.

Pearce K. A., Grimm R. H. Jr, Rao S., Svendsen K., Liebson P. R., Neaton J. D. \& Ensrud K. (1992) Population-derived comparisons of ambulatory and office blood pressures. Arch Intern Med, 152, 750-756.

Perloff D., Sokolow M. \& Cowan R. (1983) The prognostic value of ambulatory blood pressures. $7 A M A$, 249, 2792-2798.

Perloff D., Sokolow M. \& Cowan R. (1991) The prognostic value of ambulatory blood pressure monitoring in treated hypertensive patients. 7 Hypertens, 9, S33S40.

Perloff D., Sokolow M., Cowan R. M. \& Juster R. P. (1989) The prognostic value of ambulatory blood pressure measurements: further analyses. 7 Hypertens, 7, S3-S10.

Pickering T. G. \& Devereux R. B. (1987) Ambulatory monitoring of blood pressure as a predictor of cardiovascular risk. Am Heart 7, 114, 925-929.

Pickering T. G., Harshfield G. A., Kleinert H. D., Blank S. \& LaRagh J. H. (1982) Blood pressure during normal daily activities, sleep, and exercise. $7 A M A, 247$, 992-996.

Pickering T. G., Harshfield G. A., Kleinert H. D., et al. (1983) Left ventricular hypertrophy in patients with hypertension: importance of blood pressure response to regularly recurring stress. Circulation, 68, 470-476.

Pieper C., Warren K. \& Pickering T. G. (1993) A comparison of ambulatory blood pressure and heart rate at home and work on work and non-work days. 7 Hypertens, 11, 177-183.

Prisant L. M. \& Carr A. A. (1990) Ambulatory blood pressure monitoring and echocardiographic left ventricular wall thickness and mass. Am 7 Hypertens, 3, 81-89.
Rizzoni D., Muiesan M. L., Montani G., Zulli R., Calebich S. \& Agabiti-Rosei E. (1992) Relationship between initial cardiovascular structural changes and daytime and nighttime blood pressure monitoring. Am 7 Hypertens, 5, 180-186.

Takalo R., Korhonen I., Turjanmaa V., Majahalme S., Tuomisto M. \& Uusitalo A. (1994) Short-term variability of blood pressure and heart rate in borderline and mildly hypertensive subjects. Hypertension, 23, 18-24.

TurjanmaA V. (1989) Determination of blood pressure level changes in physiological situations: comparison of the standard cuff method with direct intra-arterial recording. Clin Physiol, 9, 373-387.

Turjanmaa V., Kalli S., Majahalme S., Saranummi N. \& Uusitalo A. (1987) Diurnal blood pressure profiles and variability in normotensive ambulant subjects. Clin Physiol, 7, 389-401.

Turjanmaa V. M. H., Kalli S. T. \& Uusitalo A. J. (1988) Blood pressure level changes caused by posture change and physical exercise: can they be determined accurately using a standard cuff method? 7 Hypertens, 6, S79-S81.

Turjanmaa V., Tuomisto M., Fredrikson M., Kalli S. \& Uusitalo A. (1991) Blood pressure and heart rate variability and reactivity as related to daily activities in normotensive men measured with $24-\mathrm{h}$ intra-arterial recording. 7 Hypertens, 9, 665-673.

Veerman D. P., Imholz B. P. M., Wieling W., Wesseling K. H. \& van Montfrans G. A. (1995) Circadian profile of systemic hemodynamics. Hypertension, 26, 55-59.

Verdecchia P., Porcellati C., Schillaci G., et al. (1994) Ambulatory blood pressure. An independent predictor of prognosis in essential hypertension. Hypertension, 24, 793-801.

Verdecchia P., Schillaci G., Boldrini F., Guerrieri M., Gatteschi C., Benemio G. \& Porcellati C. (1990a) Risk stratification of left ventricular hypertrophy in systemic hypertension using noninvasive ambulatory blood pressure monitoring. Am 7 Cardiol, 66, 583-590.

Verdecchia P., Schillaci G., Guerrieri M., Gatteschi C., Benemio G., Boldrini F. \& Porcellati C. (1990b) Circadian blood pressure changes and left ventricular hypertrophy in essential hypertension. Circulation, 81, 528-536.

WHO EXPERT Committee (1978) Arterial Hypertension. World Health Organization, Geneva.

Zachariah P. K., Sheps S. G. \& Moore A. G. (1990) Office blood Pressures in supine, sitting and standing positions: correlation with ambulatory blood pressure. Int 7 Cardiol, 28, 353-360. 